\title{
Coalescence aspects of III-nitride epitaxy
}

Cite as: J. Appl. Phys. 101, 054906 (2007); https://doi.org/10.1063/1.2464195

Submitted: 26 October 2006 . Accepted: 21 December 2006 . Published Online: 09 March 2007

V. Lebedev, K. Tonisch, F. Niebelschütz, V. Cimalla, D. Cengher, I. Cimalla, Ch. Mauder, S. Hauguth, O. Ambacher, F. M. Morales, J. G. Lozano, D. González, et al.

\section{ARTICLES YOU MAY BE INTERESTED IN}

Adatom diffusion at GaN (0001) and (0001) surfaces

Applied Physics Letters 73, 487 (1998); https://doi.org/10.1063/1.121909

Dislocation mediated surface morphology of GaN

Journal of Applied Physics 85, 6470 (1999); https://doi.org/10.1063/1.370150

On the origin of threading dislocations in GaN films

Journal of Applied Physics 106, 073513 (2009); https://doi.org/10.1063/1.3225920

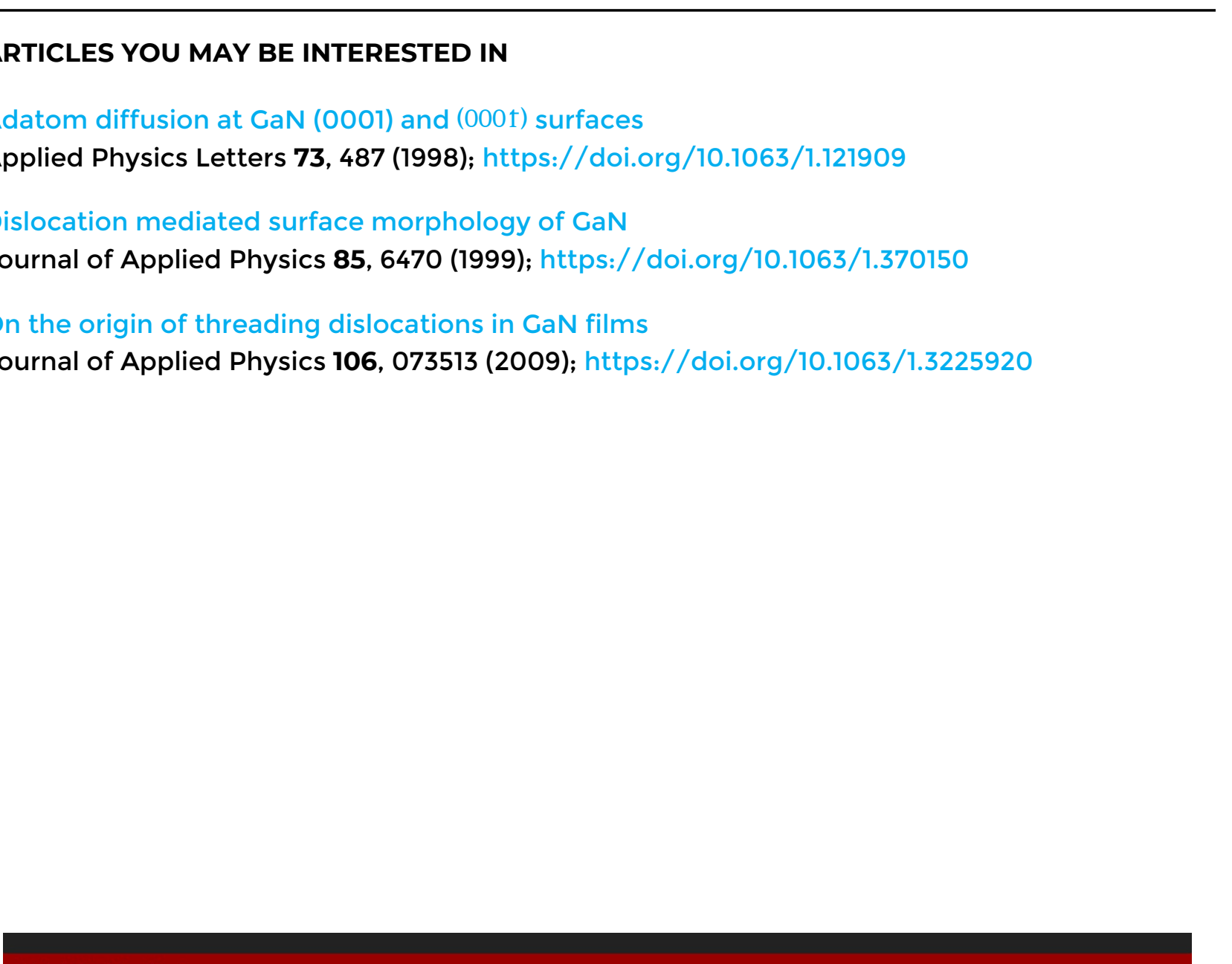

\section{HDDEN \\ Instruments for Advanced Science}

- Knowledge,

- Experience,

- Expertise

Click to view our product catalogue

Contact Hiden Analytical for further details:

w www.HidenAnalytical.com

E info@hiden.co.uk

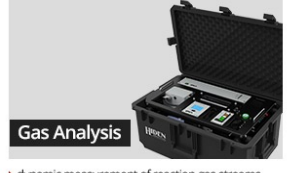

- dyramic measurement ofreaction gas streams - catalysis and thermal analys

- molecular beam studies

- fermentation, environmental and ecological studies

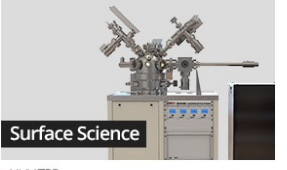

- UHVTPD

, SIMS elemental imaging -surface mapping

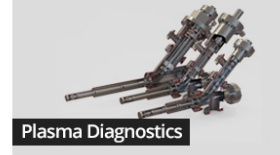

, plasma source characterization - etch and deposition process reaction mitur
View Online 


\title{
Coalescence aspects of III-nitride epitaxy
}

\author{
V. Lebedev, ${ }^{\text {a) }}$ K. Tonisch, F. Niebelschütz, V. Cimalla, D. Cengher, I. Cimalla, \\ Ch. Mauder, S. Hauguth, and O. Ambacher \\ Institute of Micro- and Nanotechnologies, Technical University Ilmenau, D-98684 Ilmenau, Germany \\ F. M. Morales, J. G. Lozano, and D. González \\ Departamento de Ciencia de los Materiales e Ingeniería Metalúrgica y Química Inorgánica, \\ Facultad de Ciencias, Universidad de Cádiz, 11510 Puerto Real-Cádiz, Spain
}

(Received 26 October 2006; accepted 21 December 2006; published online 9 March 2007)

\begin{abstract}
In this work, coalescence aspects of wurtzite-III-nitride epitaxy are addressed. The coalescence phenomena have been studied in thin epilayers by means of electron and atomic force microscopies, and electron and x-ray diffractions. This study generalizes the growth parameters responsible for the rapid coalescence of III-nitride films, and describes the coalescence qualitatively and, partly, analytically for the case of heteroepitaxy in nonequilibrium conditions. Coalescence time and the corresponding diffusion coefficients at elevated temperatures were estimated for $\mathrm{GaN}$ and $\mathrm{InN}$ depositions. The rate of coalescence has been found to impact on the structure and morphology of III-nitride epilayers. A simple growth model was suggested to explain the formation of domain boundaries and (0001) stacking faults formed during the coalescence. In particular, it is shown that two adjacent and tilted, hexagonal-shaped $2 \mathrm{H}$ domains may form a noncoherent boundary explicitly along a $\{1 \overline{1} 00\}$ plane. We also suggest that the interaction between tilted domains induces the localized lateral growth of the most epitaxially oriented domain forming a basal (0001) stacking fault followed by the formation of surface macrosteps, and consequently the termination of a threading dislocation by its dissociation and propagation under the formed (0001) stacking fault. (C) 2007 American Institute of Physics. [DOI: 10.1063/1.2464195]
\end{abstract}

\section{INTRODUCTION}

Recently, great progress in fabricating highly efficient III-nitride-based devices has been achieved. ${ }^{1}$ Extensive studies of material and device properties have been accomplished by a number of studies concerning defects and impurities, ${ }^{2,3}$ surface structures, ${ }^{4}$ and associated growth mechanisms. ${ }^{5-8}$ However, a lack of lattice-matched substrate remains the main challenge hindering the progress on the practical side. Moreover, there are still substantial problems concerning a deep understanding of the fundamental mechanisms of the III-nitride heteroepitaxy.

Epitaxial wurtzite $\left(2 \mathrm{H}_{-}\right)$III-nitride layers used for device fabrication are usually highly faulted single crystals and typically adopt a mosaic structure with subgrain boundaries delineated by threading dislocations (TDs). ${ }^{9,10}$ The origin of this structure lies on surface atomic processes under growth. It was recently shown ${ }^{11,12}$ that heteroepitaxy of III nitrides on highly mismatched substrates can be divided into four stages, each of them having a critical impact on the crystal quality of the epilayer. They are (i) three-dimensional (3D) nucleation, (ii) subsequent growth of misoriented islands in the VolmerWeber mode, (iii) partial coalescence of the islands into a continuous film, and (iv) long-term epitaxy followed by gradual improvements of the surface morphology.

$3 \mathrm{D}$ nucleation and subsequent coalescence are interesting and important problems, which control a number of physical and mechanical properties of thin films. It has been

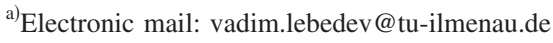

extensively studied for decades using a variety of theoretical approaches mostly based on theory of phase-ordering kinetics. ${ }^{13,14}$ However, most of the studies were devoted to computer simulations of the grain growth in the isotropic limit (i.e., when both energy and mobility of grain boundaries are isotropic), with rare attempts on anisotropy calculations. ${ }^{15,16}$ In the case of the heteroepitaxy of thin IIInitride films, both energy and mobility are strongly anisotropic, e.g., their values depend on the misorientation between two neighboring crystals and the spatial orientation of their boundaries. In addition, nonequilibrium growth conditions and the presence of liquid phase at the grain boundaries, usual for a group-III-rich growth, may also result in a strong anisotropy of both energy and mobility. Due to their complexity, either analytical or computational studies of the coalescence in III nitrides have been rarely addressed.

So far, III-nitride nanodomain coalescence has not been studied in great detail also experimentally. However, this knowledge is extremely important due to the dependence of the crystal structure on particle sizes, which further influences the performance of devices. In general the shape and size of nucleated nanocrystals depend on the crystal structure, temperature, and composition (e.g., stoichiometry). ${ }^{7}$ In many cases, the III-nitride islands have a hexagonal form with small degrees of truncation (see Fig. 1). This shape occurs since it leads to surface energy minimization for nuclei formed at equilibrium, and because of kinetics where the shape is determined by the rate at which different crystal faces grow. ${ }^{17}$ However, the combination of factors such as 

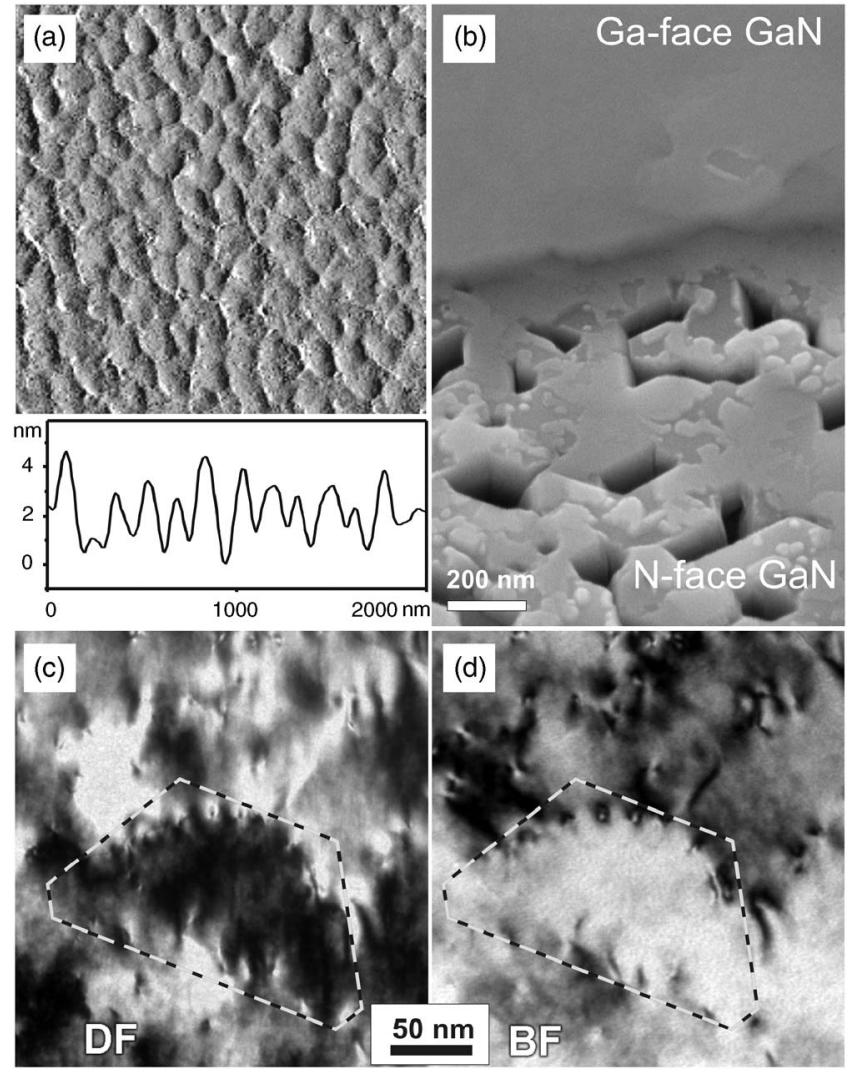

FIG. 1. Examples of the mosaic structure of III-nitride films grown on (0001) sapphire by PIMBE: (a) $2 \times 2 \mu \mathrm{m}^{2}$ AFM image of a $40 \mathrm{~nm}$ thick AlN epilayer grown at $700{ }^{\circ} \mathrm{C}$ and associated roughness profile; (b) SEM image of the lateral interface between the $\mathrm{Ga}$ and $\mathrm{N}$ faces of an $\sim 500 \mathrm{~nm}$ thick GaN(0001) LPH shows a completed coalescence on the Ga-face side and the result of a partial coalescence on the $\mathrm{N}$-face side (the surface is covered by a $10 \mathrm{~nm}$ thick Au layer for better imaging conditions); [(c) and (d)] DF (c) and BF (d) PVTEM micrographs of the same region of a $70 \mathrm{~nm}$ thick $2 \mathrm{H}$-InN layer grown on $\mathrm{GaN}(0001) /$ sapphire template. In these $2 \mathrm{~B}$ conditions using the $(11 \overline{2} 0)$ reflection near the [0001] zone axis, TDs with an edge component are visible. These images demonstrate the mosaic structure of the film and the TD distribution within the domain boundary (marked by the dashed line)

temperature, kinetics, impurities, and surface energy effects could lead to unusual nanoparticle size-shape distributions, which influences on the particle coalescence.

In order to improve growths in a systematic way it is essential to understand the underlying kinetic processes such as adsorption, desorption, and surface diffusion. In particular, adatom diffusion on the growing surfaces is considered to be a key parameter controlling the coalescence and consequently the material quality and the surface morphology. ${ }^{5}$ Recently, we have proposed ${ }^{12}$ that the coalescence process is a dominating factor controlling key crystal properties as polytype, dislocation density, and surface morphology of $2 H$-InN. The present work attempts to generalize this idea for the whole family of binary III nitrides and describe the coalescence (at least, qualitatively) for the cases of plasmainduced molecular beam epitaxy (PIMBE) and metal-organic chemical vapor deposition (MOCVD). Coalescence times and the corresponding diffusion coefficients at elevated temperatures were estimated for both cases and compared with theoretical predictions. Additionally, a simple growth model is proposed to explain the formation of domain boundaries and stacking faults during the coalescence stage.

$2 H$-AlN and $2 H$-GaN buffer layers are usually involved in the heteroepitaxial growth of III-nitride heterostructures on conventional substrates, e.g., sapphire, silicon, and silicon carbide. Consequently, the grain structure of such films plays a crucial role in the achievement of functional III-nitride devices determining the mosaic structure of the subsequent epilayers. Therefore, our discussion is extensively developed for the case of highly mismatched wurtzite heterosystems, e.g., GaN and AlN on sapphire and $\mathrm{InN}$ on AlN and GaN, having the lattice mismatch $f>10 \%$. Additionally, particular examples of AlGaN thin film deposition are also used for supporting our statements which generalize the III-N heteroepitaxy.

\section{EXPERIMENTAL DETAILS}

The MOCVD of GaN and AlN was performed at 1120 and $1190{ }^{\circ} \mathrm{C}$, respectively, at $15 \mathrm{mbar}$ pressure using a commercial Aixtron AIX200RF reactor. Triethylgallium (TEGa), trimethylaluminum (TMAl), and ammonia in hydrogen served as precursors with a V-III ratio of $\sim 2000$. The sapphire surface was cleaned in situ by etching in a hydrogen atmosphere at $1180{ }^{\circ} \mathrm{C}$. The growth was initiated by a low temperature by either a $20 \mathrm{~nm}$ thick AlN or GaN nucleation layer. Afterwards a thicker epilayer was deposited to reach the structural quality required. The detailed description of the MOCVD experiments can be found elsewhere. ${ }^{18,19}$

Other heterostructures discussed in this work were grown in a Balzer's PIMBE system with a base pressure of $\sim 1 \times 10^{-10}$ mbar. Standard Knudsen cells were used for $\mathrm{Ga}$, $\mathrm{Al}$, and In evaporations. An Oxford Applied Research radiofrequency nitrogen plasma source has been used to supply active $\mathrm{N}$ radicals from purified $6 \mathrm{~N} 5 \mathrm{~N}_{2}$ gas. The substrate temperatures were calibrated by an infrared pyrometer. The growth process was monitored by digitized patterns of reflection high-energy electron diffraction (RHEED). Molecular fluxes were monitored in situ by a quadrupole mass spectrometer and calibrated using RHEED oscillations. The PIMBE growth conditions of $2 \mathrm{H}$-AlN (Ref. 20) and $2 \mathrm{H}$ InH (Ref. 12) thin films are described elsewhere. The growth temperatures of 670 and $370{ }^{\circ} \mathrm{C}$ were used for $\mathrm{GaN}$ and $\mathrm{InN}$ depositions, respectively. In these conditions, the growth rate is proportional to the impinging group-III flux, thus getting sticking coefficients approximately equal to the unity. ${ }^{21} \mathrm{Un}-$ like that, AlN epitaxy has been performed at rather higher temperatures of $\sim 910^{\circ} \mathrm{C}$ and excess of $\mathrm{Al}$ has been used to keep the two-dimensional (2D) growth as the active mode of deposition.

For structural characterization, transmission electron microscopy (TEM) was performed in electron microscopes operating at $200 \mathrm{keV}$ [TECNAI 20S-TWIN (FEI) and JEM2011 from JEOL]. Mechanical thinning and ion milling were used to prepare specimens for cross-section TEM (XTEM) and plan-view FEM (PVTEM) inspection. Electron diffraction patterns and micrographs obtained in bright-field $(\mathrm{BF})$ 
and dark-field (DF) modes by conventional two-beam (2B) conditions and high resolution TEM (HRTEM) were obtained and analyzed.

Structural analysis was also performed by high resolution $\mathrm{x}$-ray diffraction (XRD) using a Bruker D8 diffractometer. Rocking curves at the symmetric (0002) and reciprocal space maps at the symmetric (0002) and the asymmetric $(20 \overline{2} 5) 2 H$ reflections were taken to evaluate the crystal quality and the residual strain in the III-nitride epilayers, respectively.

\section{RESULTS}

\section{A. Mosaic structure of III-nitride epilayers}

So far, heteroepitaxial thin $2 \mathrm{H}$-III-nitride layers are characterized by a mosaic structure with an average subgrain size of $50-250 \mathrm{~nm}$ arising as the result of initial threedimensional (3D) nucleation and partial coalescence of the islands. Moreover, a mean grain size has been found to be a function of the growth conditions and the mismatch strain induced in the heterosystem representing the wetting properties of the epilayer deposited onto the substrate. In this part, some examples of the mosaic structures of thin binary IIInitride films will be considered to provide characteristic domain sizes for the numerical estimations and the forthcoming discussion.

In Fig. 1, atomic force microscopy (AFM), scanning electron microscopy (SEM), and PVTEM micrographs of PIMBE III-nitride epilayer surfaces exhibiting a characteristic domain structure are presented. AFM image in Fig. 1(a) shows a mosaic surface of a $40 \mathrm{~nm}$ thick $2 \mathrm{H}$-AlN layer nucleated on (0001) sapphire at $700{ }^{\circ} \mathrm{C}$ and annealed at $1100{ }^{\circ} \mathrm{C}$ for $2 \mathrm{~min}$. Typical layers have pronounced grain structures with the mean grain size of $\sim 100 \mathrm{~nm}$ and the root mean square (rms) roughness of $\sim 1.5 \mathrm{~nm}$.

In Fig. 1(b), SEM micrograph of the lateral interface between $\mathrm{Ga}$ and $\mathrm{N}$ faces of an $\sim 500 \mathrm{~nm}$ thick $\mathrm{GaN}(0001)$ lateral polarity heterostructure ${ }^{22}(\mathrm{LPH})$ shows a complete coalescence on the Ga-face side and the result of a partial coalescence on the N-face side. Due to the large GaN/ sapphire lattice mismatch and a reduced diffusion of $\mathrm{Ga}$ atoms on the $\mathrm{N}$-face surface the coalescence of the $\mathrm{N}$-face domains cannot be completed, leading to the specific mosaic structure of the film grown directly on sapphire with an average grain size of $\sim 250 \mathrm{~nm}$.

Figures 1(c) and 1(d) show PVTEM images of a $70 \mathrm{~nm}$ thick $2 H$-InN layer grown on $\mathrm{GaN}(0001)$ template demonstrating the partial coalescence between in-plane misoriented $\mathrm{InN}$ domains having the mean grain size ranging from 50 to $100 \mathrm{~nm}$. It was demonstrated elsewhere ${ }^{12}$ that the lateral interaction of such domains leads to the formation of the $\mathbf{b}=1 / 3\langle 11 \overline{2} 3\rangle$ TDs distributed along the domain boundary with a spacing ranging from 20 to $50 \mathrm{~nm}$.

In contrast to PIMBE, MOCVD is capable of providing nucleation epilayers having larger domains. In Fig. 2, 5 $\times 5 \mu \mathrm{m}^{2}$ AFM images demonstrate an evolution of the mean grain size with respect to the epilayer thickness for 70,140 , and $210 \mathrm{~nm}$ thick GaN epilayers grown by MOCVD on $\operatorname{AlN}(20 \mathrm{~nm}) / \mathrm{sapphire}$ templates. The gradual increase of the

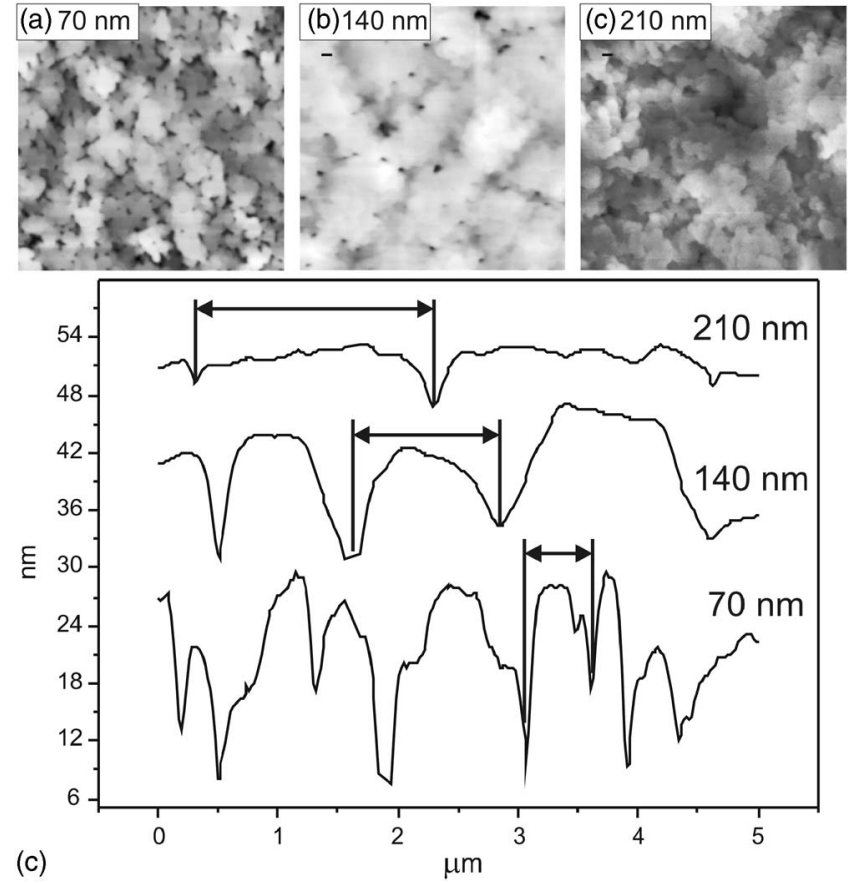

FIG. 2. $5 \times 5 \mu \mathrm{m}^{2}$ AFM images of (a) 70, (b) 140 , and (c) $210 \mathrm{~nm}$ thick $\mathrm{GaN}$ epilayers grown by MOCVD on a $20 \mathrm{~nm}$ thick AlN nucleation layer deposited on (0001) sapphire. (d) The surface profiles extracted from the AFM images.

characteristic domain size from $300 \mathrm{~nm}$ to $\sim 2 \mu \mathrm{m}$ for 70 and $210 \mathrm{~nm}$ thick epilayers, respectively, corresponds to the long-term coalescence process of the nucleated islands into the continuous single crystalline film.

The mosaic structure of AlN layers grown by MOCVD and PIMBE on sapphire was compared elsewhere. ${ }^{23}$ In particular, it was shown that the characteristic mean grain size depends on the deposition technology used, being a function of actual growth conditions provided by the specific method, i.e., deposition temperature, arrival flux of the species, group-III/group-V flux ratio, etc. The epitaxy of AlN thin films results in domain sizes of $80-120 \mathrm{~nm}$ and of 150-300 nm for PIMBE and MOCVD methods, respectively. In both cases, however, the mean domain size is limited by both kinetics and thermodynamics of the heterogeneous crystal growth addressed in the Discussion section.

\section{B. Coalescence dynamics}

\section{GAN/sapphire and GaN/AIN(0001) heterosystems}

To study the dynamics of the coalescence in detail, a selective MOCVD of Ga-face $\mathrm{GaN}(0001)$ nanostripes has been carried out on nanopatterned $\mathrm{Al}_{2} \mathrm{O}_{3}(0001)$ substrates. $100 \mathrm{~nm}$ wide and $10 \mu \mathrm{m}$ long nanogrooves have been created in $\mathrm{Si}_{x} \mathrm{~N}_{y} / \mathrm{Al}_{2} \mathrm{O}_{3}$ templates by using e-beam lithography and reactive ion-etch techniques. The grooves were prepared along $\langle 11 \overline{2} 0\rangle$ and $\langle 10 \overline{1} 0\rangle$ directions of the sapphire surface to study the coalescence on the orthogonal facets. The etch process parameters were chosen to establish dashed-trench geometry of the grooves. In Fig. 3(a), it is shown that the etched grooves are not homogeneous in depth and, as a result, functional windows are opened in the $\mathrm{Si}_{x} \mathrm{~N}_{y}$ mask providing circular spots of open sapphire surface. These func- 


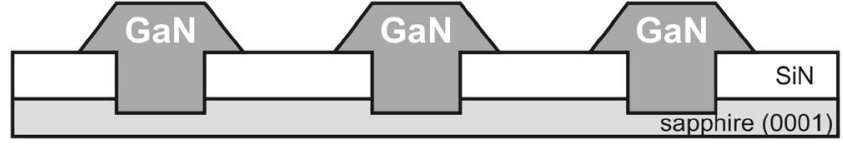

1. SiN-mask

2. nucleation

3. coalescance
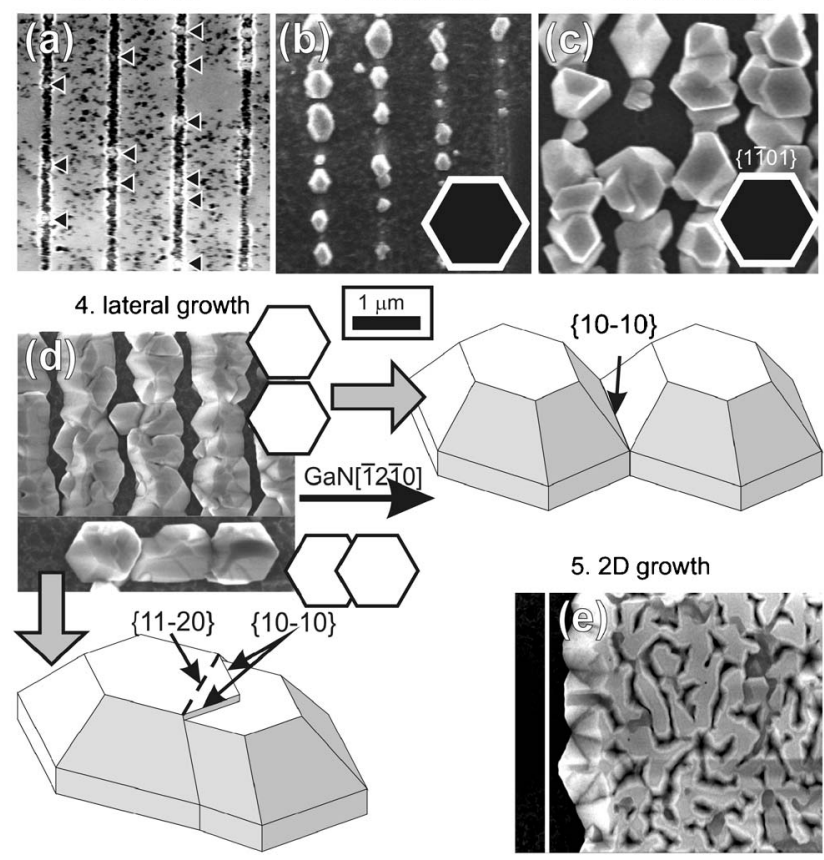

5. 2D growth

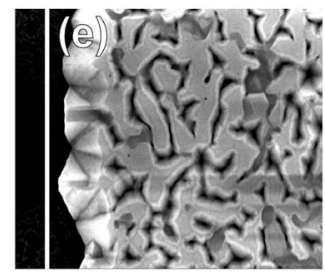

FIG. 3. SEM micrographs of (a) a nanopatterned $\mathrm{Si}_{x} \mathrm{~N}_{y} /$ sapphire template (the triangles point to the open sapphire surface) and [(b)-(d)] various stages of nucleation and coalescence of $2 \mathrm{H}$-GaN domains during the selective MOCVD. The Draws in (d) show the characteristic shape and interaction planes for GaN nuclei. (e) A fragment of a large reference window used for 2D nucleation.

tional windows with a diameter of $\sim 50-60 \mathrm{~nm}$ are ideal nucleation sites for single $\mathrm{GaN}$ isolated islands-as it was discussed above; the thin GaN layers grown by MOCVD have a domain structure with domain sizes of 200-300 nm. The characteristic dimensions of the nucleation spots ensure that homogeneous, single islands will be formed at every nucleation point. Moreover, the short average distance between these spots allows growing GaN domains to coalesce within a short time of deposition.

Figure 3 shows various stages of nucleation and coalescence of $2 \mathrm{H}-\mathrm{GaN}$ domains during the deposition. An estimation of the coalescence time $\left(\tau_{C}\right)$ for islands of average radius of $\sim 200 \mathrm{~nm}$ yields a value of about $90 \mathrm{~s}$, if we assume that the coalescence starts in a state similar to that shown in Fig. 3(b) and is completed when a total junction between nuclei happens along grooves as shown in Fig. 3(d).

The driving forces for the high rate of lateral growth and, consequently, of the coalescence are a surface diffusion of the adsorbed species. Adatoms diffuse on the island surface from the regions of high curvature (fewer neighbors and therefore less strongly bound) towards the regions of lower curvature (e.g., facets), providing lateral growth of the initial nuclei. ${ }^{17}$ It is necessary to note that used pseudo-onedimensional growth geometry simplifies significantly the physical picture of the coalescence. The artificial structures used allow studying the coalescence in both orthogonal directions $(\langle 11 \overline{2} 0\rangle$ and $\langle 10 \overline{1} 0\rangle)$ of the hexagonal structure separately [see Fig. 3(d)].

Macroscopic theories of coalescence via atomic surface diffusion predict a coalescence time, ${ }^{24}$

$$
\tau_{c}=\frac{k T}{C D_{s}(T) \gamma}\left(\frac{R}{a}\right)^{4},
$$

where $R \sim 250 \mathrm{~nm}$ is the domain radius, $\gamma$ is the surface energy of $\mathrm{GaN}(0001), C \approx 25$ is a numerical constant, $D_{s}(T)$ is the surface diffusion coefficient, $T=1120^{\circ} \mathrm{C}$ is the growth temperature, and $a \sim 0.3 \mathrm{~nm}$ is the atomic dimension. The surface energies of the minimum energy GaN are nearly equal for various surfaces of GaN: $\gamma(11 \overline{2} 0) \sim 123 \mathrm{meV} / \AA^{2}$, $\gamma(10 \overline{1} 0) \sim 110 \mathrm{meV} / \AA^{2}, \quad$ and $\quad \gamma(0001) \sim 125 \mathrm{meV} / \AA^{2} .^{25}$ Thus, we assumed $\gamma=1.89 \mathrm{~J} / \mathrm{m}^{2}$ for the whole island surface area.

For the case shown in Fig. 3 (Ga-face GaN) we obtain $D_{s}\left(1120^{\circ} \mathrm{C}\right)=2.2 \times 10^{-12} \mathrm{~m}^{2} \mathrm{~s}^{-1}$ using the experimental value of $\tau_{c}=90 \mathrm{~s}$. Thus, the estimated distance that an atom travels within the coalescence time $\tau_{c}$ is $D_{\mathrm{dif}}=\left(D_{s} \tau_{c}\right)^{1 / 2}$ $\sim 14 \mu \mathrm{m}$ which is much higher than the particle size after the coalescence. Additionally, the observed flattop pyramids indicate a high lateral growth rate. Such a shape favors a rapid coalescence and a flatter morphology of the thicker films. ${ }^{17}$ One can see it in Fig. 3(e), where a larger window was used as a nucleation test area for a conventional 2D coalescence.

In these series of experiments, it was also observed that the grain boundaries between interacting $\mathrm{GaN}(0001)$ islands are predominantly formed along the $\{1 \overline{1} 00\}$ planes [Fig. $3(d)]$. The analyses of the controlled coalescence process do not indicate that the hexagonal-shaped domains interact by the $\{11 \overline{2} 0\}$ planes. The domain interaction on this plane family is rather poor, leading to the degeneration of the $\{11 \overline{2} 0\}$ contact interfaces to the grain boundaries along the $\{1 \overline{100}\}$ plane [Fig. 3(d)]. This can be explained by the fact that boundaries along the $\{1 \overline{100}\}$ planes have the lowest energies as well as the lowest mobilities. ${ }^{25,26}$ Therefore, energy minimization would make nuclei tend to bond by low-energy boundaries. $^{15,16}$

Additionally, to compare the coalescence of $\mathrm{Ga}$ - and N-face films directly, GaN LPHs (Ref. 22) were grown on patterned $2 \mathrm{H}$-AlN/sapphire(0001) templates by PIMBE. The LPHs contained a periodic lateral structure consisting of $\sim 100 \mu \mathrm{m}$ wide Ga- and N-face GaN stripes. The lateral interface between two faces is shown in SEM and AFM images [Fig. 1(b) and Figs. 4(a) and 4(b)]. Here, the Ga-face $\mathrm{GaN}(0001)$ has been grown on the developed AlN stripes (lattice mismatch $f<1 \%$ ), while the $\mathrm{N}$-face $(000 \overline{1})$ layer was nucleated directly on the sapphire surface $(f \sim 14 \%)$. As a result of lateral interaction of inverted domains, inversion domain boundaries (IDBs) are formed at vertical interfaces between two phases of $2 \mathrm{H}-\mathrm{GaN} .^{22}$ The GaN epitaxy was initiated at $\sim 650{ }^{\circ} \mathrm{C}$, which is a typical growth condition for the Ga-face material. As one can see in Figs. 1(b) and 4, due to the large mismatch between sapphire and GaN as well as 

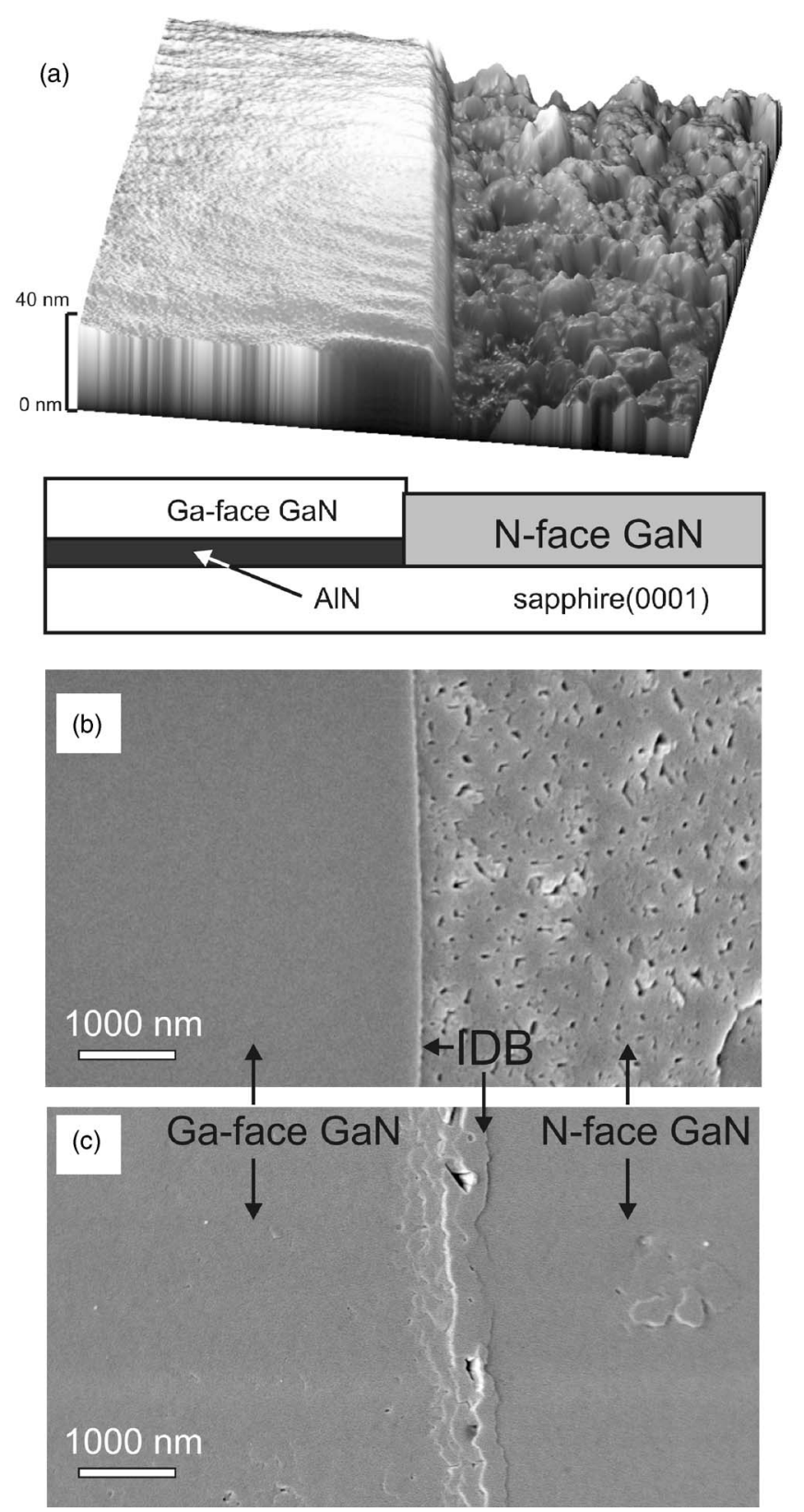

FIG. 4. $3 \times 3 \mu \mathrm{m}^{2}$ AFM (a), and SEM [(b) and (c)] images of the GaN LPH demonstrating the impacts of surface diffusion and of low-temperature buffer layer on the morphology of $\mathrm{N}$-face polarity $2 \mathrm{H}$-GaN stripes.

due to the low mobility of $\mathrm{Ga}$ adatoms on the $\mathrm{N}$-face $\mathrm{GaN},{ }^{5,21,27,28}$ the N-face side exhibits a very faceted growth surface with the mean grain size of $\sim 200 \mathrm{~nm}$, while Ga-face stripes are single-crystalline, smooth films.

\section{InN/GaN(0001) heterosystem}

Figure 5 shows an initial stage of nucleation and partial coalescence of $2 \mathrm{H}$-InN domains during the deposition on $\mathrm{GaN}(0001)$ by $\mathrm{PIMBE}$ at $370^{\circ} \mathrm{C}(f \sim 13 \%)$. An estimation of the coalescence time $\left(\tau_{C}\right)$ for islands of average diameter of $\sim 60 \mathrm{~nm}$ yields a value of about $35 \mathrm{~s}$. For the case shown in Fig. 5 we obtain very low $D_{s}\left(370^{\circ} \mathrm{C}\right) \sim 6 \times 10^{-16} \mathrm{~m}^{2} \mathrm{~s}^{-1}$ using the experimental value of $\tau_{c}=35 \mathrm{~s}$. The estimated distance that an atom travels within the coalescence time $\tau_{c}$ is $D_{\text {dif }} \sim 0.14 \mu \mathrm{m}$, which is only slightly larger than the aver-
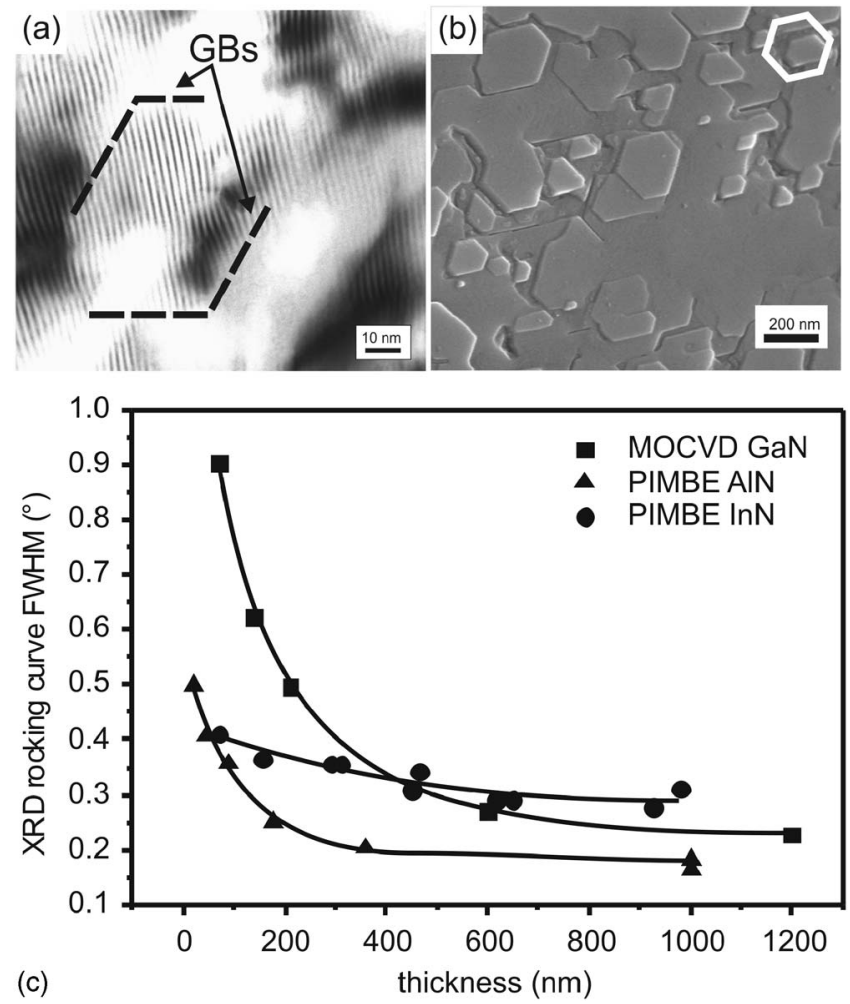

FIG. 5. [(a) and (b)] The mosaic structure of $2 H-\mathrm{InN}$ grown on GaN/ sapphire by PIMBE: (a) the PVTEM image of a $10 \mathrm{~nm}$ thick $\mathrm{InN}$ layer shows a typical domain size of $\sim 50 \mathrm{~nm}$. The moiré contrast is due to the overlapping of the InN and $\mathrm{GaN}$ related atomic planes. (b) SEM image of a $70 \mathrm{~nm}$ thick InN layer after the partial coalescence stage. (c) FWHM of XRD rocking curves taken at the (0002) reflex measured on MOCVD GaN, and PIMBE InN and AlN samples as a function of the epilayers thickness.

age crystal size after the coalescence. In this particular case, a small mean grain size of the nucleated domains as well as a partial character of the coalescence are determined by low temperature of the deposition. The latter is illustrated by the SEM micrograph of a $70 \mathrm{~nm}$ thick $\mathrm{InN}$ epilayer shown in Fig. 5(b). For this sample, the coalescence is not completed and the surface structure is still dominant by large hexagonal domains with an average size of $\sim 200 \mathrm{~nm}$.

A partial character of the coalescence results in slow improvements of the material quality with increasing thickness of the epilayer. In Fig. 5(c), the full width half maximum (FWHM) of the XRD rocking curves measured on IIInitride epilayers is plotted as a function of the epilayers thickness. Among the selected systems, InN on GaN shows the lowest rate of crystal quality improvements reflecting specific conditions of the InN growth, i.e., low growth temperature of In-face $\mathrm{InN}$, which is limited by the thermal decomposition of $\mathrm{InN}$ at $\sim 500{ }^{\circ} \mathrm{C}$ in vacuum.

\section{AIN/sapphire heterosystem}

$2 \mathrm{H}$-AlN buffer layers are commonly used in the heteroepitaxial growth of III-nitride heterostructures on conventional substrates. It was found that the heteroepitaxial growth across the III-nitride/sapphire interface is dictated by the $\mathrm{Al}-\mathrm{O}$ bonding, which results in a $30^{\circ}$ in-plane rotation of the nitride lattice with respect to the sapphire substrate. ${ }^{29}$ It delineates the epitaxial relationship between the film and sub- 
strate as follows: $(0001)_{f}\left\|(0001)_{s},[01 \overline{1} 0]_{f}\right\|[\overline{1} 2 \overline{1} 0]_{s}$, and $[\overline{1} 2 \overline{1} 0]_{f} \|[01 \overline{1} 0]_{s}$. The $30^{\circ}$ rotation results in a mismatch of about $12 \%$ between the substrate $\left(a_{s} / \sqrt{ } 3\right)$ and the film $\left(a_{f}\right)$. The large lattice mismatch is accommodated by domain matching of the lattice constants $\left(7 a_{s} / \sqrt{ } 3=6 a_{f}\right)$, where seven $(\overline{1} 2 \overline{1} 0)$ planes of sapphire match six $(01 \overline{1} 0)$ planes of the AlN film. ${ }^{30}$

In general, the quality of the AlN films grown directly (i.e., without a low-temperature buffer layer) on any plane of sapphire is poor as a result of the large lattice mismatch and difference in the coefficients of thermal expansions between the epilayer and the substrate. A low-temperature growth of AlN $\left(<700{ }^{\circ} \mathrm{C}\right.$ for PIMBE) allows full coverage of the substrate to be quickly achieved, and the subsequent layers can be grown at the optimal pseudohomoepitaxial conditions. ${ }^{31} \mathrm{It}$ was also shown that the properties of the subsequent epilayers are strongly influenced by the thickness and growth temperature of the buffer layer.

\section{Effect of a low-temperature nucleation layer}

Any kind of deposition of III-nitride epilayers yields low-energy-deposited particles, which preserve their integrity upon impact onto the substrate at high temperatures. To overcome this repulsion, an initiation of the growth at low temperatures is widely used in III-nitride epitaxy resulting in formation of highly disordered (textured) "buffer" layers. In this stage, neither coalescence nor ripening processes occur. The growth of such layer can overcome the problems related to the high lattice mismatch ${ }^{32}$ and even to the differences in the crystal cell symmetry. ${ }^{33,34}$ It allows a full coverage of the substrate surface, but leads to a coarsening of the growing layer [see Fig. 1(a)]. The low-temperature deposition is usually followed by annealing and long-term epitaxy at higher temperatures to achieve crystal quality and surface morphology suitable for device applications. Obviously, the temperature increase leads to the coalescence of the deposited textured aggregate.

One illustration of this phenomenon is the time evolution of RHEED patterns during PIMBE nucleation and growth of AlN(0001) on a $\mathrm{Si}(111)$ substrate (Fig. 6). The film was nucleated with the growth rate of $\sim 0.5 \mathrm{ML} \mathrm{s}^{-1}$ (ML denotes monolayer) at a low temperature of $\sim 700{ }^{\circ} \mathrm{C}$ and at a high Al supersaturation [Fig. 6(a)]. Then, after a short time of $\sim 60 \mathrm{~s}$ of nucleation, the substrate temperature was increased rapidly $\left(50{ }^{\circ} \mathrm{C} / \mathrm{min}\right)$ till the growth point at $\sim 910{ }^{\circ} \mathrm{C}$ [see Figs. 6(b) and 6(c)]. As one can see in these RHEED patterns, the nucleated film consists of highly misoriented nuclei of $2 \mathrm{H}$ polytype at the beginning of the process. A mean degree of the tilt is as high as $20^{\circ}$ [volume angle corresponding to an angular width of the arc shown in Fig. 6(a)]. If the growth is continued at $910^{\circ} \mathrm{C}$, a well developed, "streaky" pattern is formed after approximately $50 \mathrm{~nm}$ of material deposition indicating a completed coalescence of the islands. In this case, both low diffusion and relatively high deposition rates increase, resulting in a high $2 \mathrm{H}$-AlN nuclei density at low temperature. Additionally, it is generally assumed that the evolution of the average grain area obeys the time power law growth $\langle A\rangle \sim t^{k} .{ }^{13}$ In accordance with theoretical
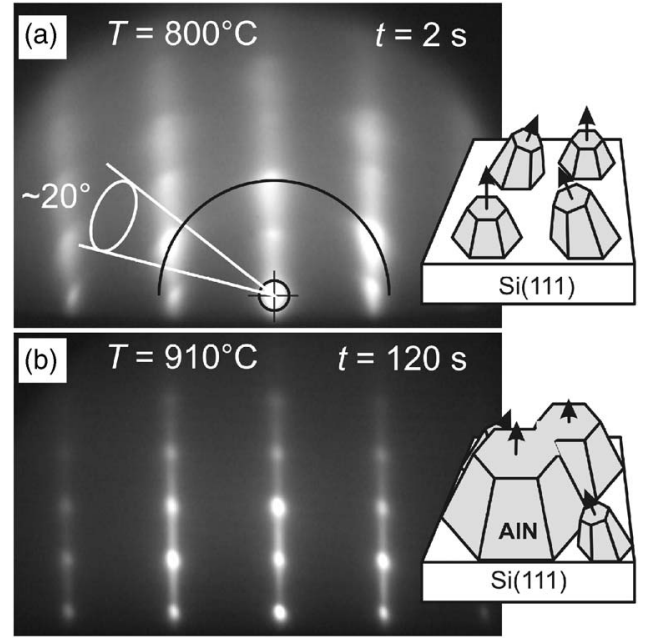

$t=120 \mathrm{~s}$

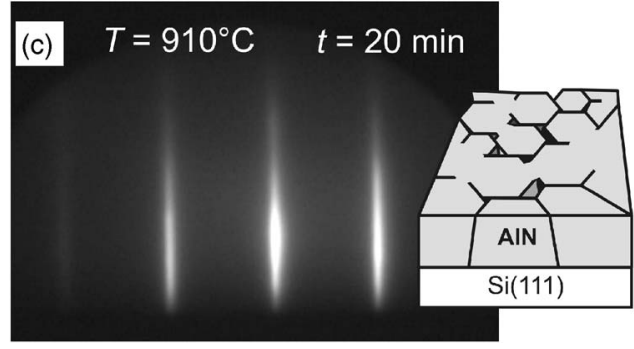

FIG. 6. RHEED pattern time evolution for PIMBE of $2 H$-AIN(0001) film nucleated on $\mathrm{Si}(111)$ surface: (a) nucleation of the slightly misoriented islands, (b) coalescence of the islands into the continuous film at the elevated temperature, and (c) long-term 2D AlN epitaxy.

predictions, ${ }^{16}$ in the case of highly textured polycrystalline aggregates, the average area of the grains is growing linearly with time $(k \sim 1)$, allowing a full coverage of the substrate surface to be quickly achieved. A subsequential increase in the temperature leads to the partial coalescence of the domains into a continuous film. In fact, after $\sim 10 \mathrm{~nm}(2 \mathrm{~min})$ of a high-temperature $\left(910{ }^{\circ} \mathrm{C}\right)$ growth, the surface gets rough but single crystalline indicating a partially completed coalescence and a pseudo-2D growth [Fig. 6(b)]. A longterm epitaxy at $910{ }^{\circ} \mathrm{C}$ results in a smoother surface [Fig. 6(c)] with a rms roughness of $\sim 1 \mathrm{~nm}$ as measured by AFM.

The impact of coalescence on the crystal quality of AlN epilayers is shown in Fig. 6(c). The crystal quality of the layers of variable thickness has been evaluated by means of XRD rocking curves reflecting angular misorientation of the islands in [0001] direction. One can see that the maximum rate of reduction of the FWHM occurs in the range of $50-100 \mathrm{~nm}$ corresponding to the active phase of the coalescence process.

Another example of the effect of the low-temperature buffer on material quality is the growth of GaN LPHs by PIMBE. In Fig. 4(c), the SEM image of GaN LPH grown on a low-temperature GaN buffer layer is shown. The obvious improvements in the $\mathrm{N}$-face material morphology are the result of a $40 \mathrm{~nm}$ thick GaN buffer layer deposited at $\sim 500{ }^{\circ} \mathrm{C}$, which ensures a full coverage of the sapphire surface. The initial deposition is followed by the annealing step at $650{ }^{\circ} \mathrm{C}$, leading to the improvements of the $\mathrm{GaN}$ crystal quality due to the island coalescence. In turn, the subsequent deposition at high temperature improves the surface morphology. 
TABLE I. Primary and residual strains in the investigated heterosystems.

\begin{tabular}{lccc}
\hline \hline \multicolumn{1}{c}{ Heterosystem } & $\begin{array}{c}\text { Primary } \\
\text { strain } \\
(\%)\end{array}$ & $\begin{array}{c}m: n \\
\text { ratio }\end{array}$ & $\begin{array}{c}\text { Residual } \\
\text { strain } \\
(\%)\end{array}$ \\
\hline $\mathrm{AlN} / \mathrm{Si}(111)$ & 23 & $5: 4^{\mathrm{a}}$ & $1.3^{\mathrm{a}}$ \\
$\mathrm{GaN} / \mathrm{Al}_{2} \mathrm{O}_{3}$ & 16 & $7: 8^{\mathrm{a}}$ & $\sim 2^{\mathrm{b}}$ \\
$\mathrm{AlN} / \mathrm{Al}_{2} \mathrm{O}_{3}$ & 13 & $6: 7^{\mathrm{a}}$ & $\sim 2^{\mathrm{b}}$ \\
$\mathrm{Al}{ }_{0.3} \mathrm{Ga}_{0.7} \mathrm{~N} / \mathrm{GaN}$ & 0.8 & $1: 1^{\mathrm{c}}$ & $0.8^{\mathrm{c}}$ \\
$\mathrm{InN} / \mathrm{GaN}$ & 11 & $8: 9^{\mathrm{a}}$ & $\sim 4^{\mathrm{b}}$ \\
$\mathrm{InN} / \mathrm{AlN}$ & 13 & $7: 8^{\mathrm{a}}$ & $\sim 4^{\mathrm{b}}$ \\
\hline \hline
\end{tabular}

${ }^{\mathrm{a}}$ HRTEM, see Refs. 12, 32, 35, and 36.

${ }^{\mathrm{b}}$ In situ RHEED, see Ref. 12 for method description.

${ }^{\mathrm{c}}$ HRTEM, this work.

\section{DISCUSSION}

Despite the differences in mechanical properties, primary mismatch strains, and deposition methods used, all the investigated hexagonal heterosystems show nearly similar initial surface morphology and dynamics of the material quality improvements. It indicates that the residual misfits remaining in the heterosystems after nucleation stage do not differ significantly for the heterosystems discussed above.

In highly mismatched hexagonal systems, it is generally not possible to strain the film lattice elastically to fit onto the template lattice at the interface. Instead there is an approximate matching of $m(h k l)_{f}$ planes of the film with $n(h k l)_{s}$ planes of the template with $m=n+1$ for $f<0$, where $(h k l)_{f}$ is parallel to $(h k l)_{s}$ and both sets are normal to the interface. ${ }^{36}$ Since $m=n \pm 1$, there is an extra half plane either in the film (for $f<0$ ) or in the substrate (for $f>0$ ). Namely, these planes define a geometrical misfit dislocation (GMD), a $60^{\circ}$ dislocation with Burgers vector $\mathbf{b}$ and line parallel to $\langle 2 \overline{1} \overline{1} 0\rangle$ directions and lying on the (0001) basal plane, which exists in a high percentage from the very start of the film growth. In the discussed cases, GMDs are the only mechanism to relief a huge primary strain in the heterosystem down to the value allowing epitaxial growth. In Table I, the values of the primary and residual in plane strains for the investigated systems are listed. One can see that for the majority of the heterosystems residual misfit does not exceed $1.5 \%$, while $2 \mathrm{H}$-InN epilayers grown on $\mathrm{GaN}$ and AlN templates show the highest residual strain of $\sim 4 \%$ measured dynamically by in situ RHEED. ${ }^{12}$ In particular, this fact explains a low rate of improvements in the material quality of InN epilayers represented by the FWHM versus thickness curves shown in Fig. 5(c).

\section{A. The origin of the mosaic structure}

Besides the accommodation of the initial misfit by introduction of GMDs at the heterointerface, ${ }^{12,32}$ there is another way to relax misfit strain within the nucleated epilayer destabilizing the growth front. ${ }^{11}$ Indeed, in the simplified case, the wetting conditions for the epilayer are defined by the balance between the formation energies of the film surface $\sigma_{\text {film }}$, the template surface $\sigma_{\text {sub }}$, and the formation energy of the epilayer $E_{f},{ }^{37}$ which is a function of the actual growth
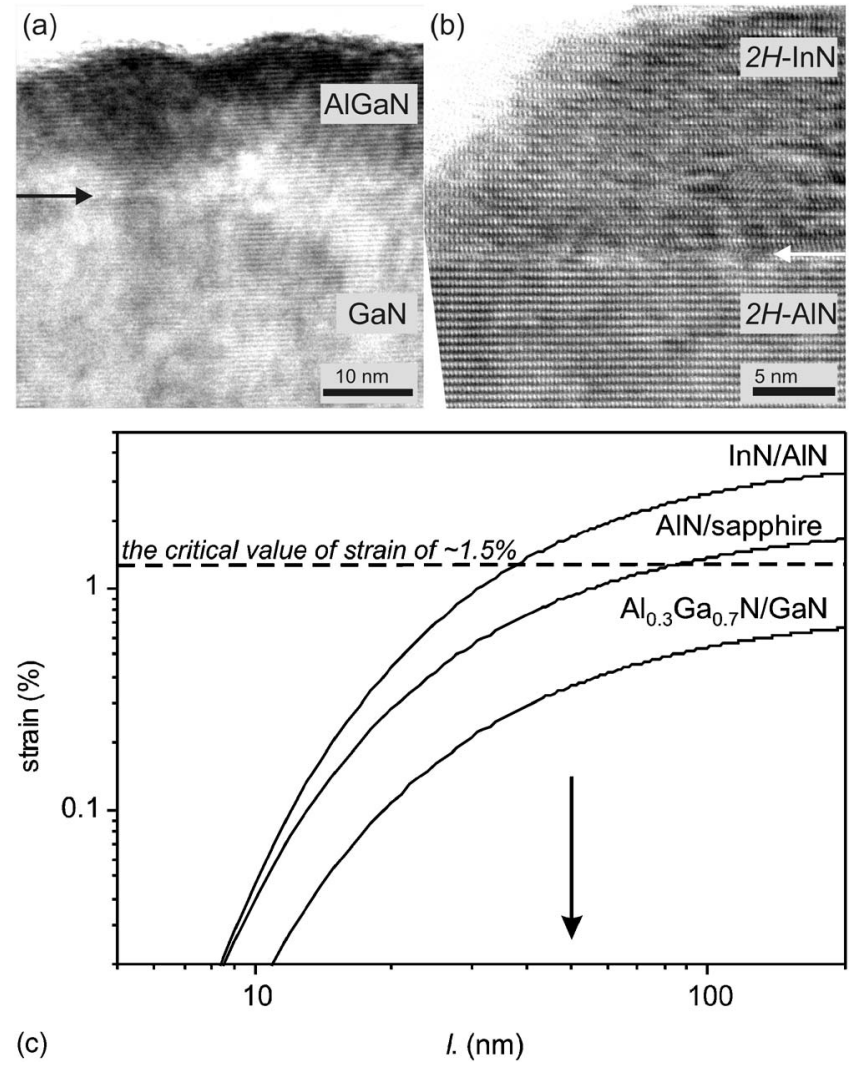

FIG. 7. HRTEM images (a) $\mathrm{Al}_{0.3} \mathrm{Ga}_{0.7} \mathrm{~N} / \mathrm{GaN}$ hetorostructure and (b) of $2 \mathrm{H}$-InN island nucleated on an AlN template; (c) calculated in-plane strain due to the island growth as a function of the characteristic island size $l$ for different $2 H$ heterosystems.

conditions (e.g., the supersaturation $S$ and temperature $T$ ) and the stress energy $E_{\text {str }}$ arising from the mismatch between template and epilayer,

$$
E_{f}\left(S, T, E_{\text {str }}\right)>\sigma_{\text {film }}-\sigma_{\text {sub }}-\Delta E .
$$

In other words, the residual misfit strain can destabilize the growth front by producing surface modulation ${ }^{20}[$ Fig. 7(a) $]$ or eventually forming islands [Fig. 7(b)]. ${ }^{12}$ The last term in Eq. (2), $\Delta E$, represents the reduction in the total energy of the system due to such pseudoperiodical undulations at the growing surface. ${ }^{20,37}$ Consequently, the nucleation step can result in a second-order elastic relaxation of the epilayer within the nucleated nanoislands.

As an example, we will compare three heterosystems grown by PIMBE representing the boundary cases for the wurtzite III nitrides. After initial misfit relaxation via GMDs, $\mathrm{InN} / \mathrm{AlN}$ and $\mathrm{AlN} / \mathrm{Al}_{2} \mathrm{O}_{3}$ heterosystems have residual strains of $\sim 4.1 \%$ and of $\sim 2 \%$, respectively, as it was shown by in situ RHEED and HRTEM observations. ${ }^{12}$ In contrast, $\mathrm{Al}_{0.3} \mathrm{Ga}_{0.7} \mathrm{~N}$ layer grown on $\mathrm{GaN}$ pseudomorphically has to accommodate the residual strain of $\sim 0.8 \%$.

Approximated analytical solutions for the equilibrium strains have been discussed in the literature for a periodic set of thin deposits. ${ }^{11,12,38}$ It was shown that the surface stress may enhance, compensate, or decrease the epitaxial misfit. Moreover, the island relaxes by its free borders so that the lateral stress becomes height dependent. In the frame of a 2D thick ribbon model, ${ }^{38}$ a simple isotropic approximation for 
the relaxation of the topmost layers of the $2 \mathrm{H}$ islands can be obtained. The model assumes that the strain in an infinitely long ribbon of height $h$ and width $l$, deposited coherently on a substrate having a misfit $f_{0}$, is given by

$$
\begin{aligned}
& \mathfrak{I}_{y y}(x, N)=\mathfrak{I}_{y y}(x, 0)\left\langle M_{1}\right\rangle^{N-1}, \\
& \left\langle M_{1}\right\rangle=1-\frac{2 \pi a}{l}+\left(1+\sqrt{\frac{2 \pi a}{l}}\right)^{2} \exp \left(-\sqrt{\frac{2 l}{a \pi}}\right),
\end{aligned}
$$

where $N$ defines the number of monolayers and varies from 0 to $h / a$ - the total number of monolayers included in the ribbon of height $h$.

The dependence of the residual strain remaining in the system on the characteristic island width $l$ based on Eq. (3) is presented in Fig. 7(c). Using this approximation, we can interpret the experimental results obtained by TEM (Fig. 7) in the following way. One can assume a critical value of the residual strain, $f_{c}$, which is a boundary between an island and continuous film growth. The value of $f_{c}$ depends on the elastic stiffness constants of the deposit and the actual growth parameters (e.g., supersaturation). However, the typical experimental values of $f_{c}$ lie in the range of $1 \%-2 \%$. Accepting $f_{c} \sim 1.5 \%$ as a boundary between the $2 \mathrm{D}$ and $3 \mathrm{D}$ growth modes, we can confirm that for the $\mathrm{Al}_{0.3} \mathrm{Ga}_{0.7} \mathrm{~N} / \mathrm{GaN}$ system, $l$ is infinitely large providing continues film growth. The residual strain relief is achieved by destabilization of the growth front resulting in the periodical undulations of the growing surface [Fig. 7(a)]. On the other hand, PIMBE of the InN/AlN and $\mathrm{AlN} / \mathrm{Al}_{2} \mathrm{O}_{3}$ heterosystems can be stabilized only by the reduction of the characteristic domain size resulting in the formation of the mosaic structure of the film with $l$ of $\sim 50-100 \mathrm{~nm}$. These values correspond well to the experimentally observed characteristic size of the III-nitride islands taking part in the subsequent coalescence.

According to the suggested model, the residual strain in the epilayer determines the characteristic size of the growing islands preventing the growth of large area domains during the nucleation stage. However, two important notes concerning the model restrictions have to be made. The model does not take into account a plastic deformation of the islands as well as kinetic parameters governing island growth. ${ }^{11}$ Since surface stresses are adsorption sensitive, a surface adsorption may force the equilibrium strains and stresses to change in a reversible fashion. The latter is the case for MOCVD grown III-nitride films, where significantly larger domain sizes governed by high pressures and high temperatures characteristic for the growth can be reached.

\section{B. Coalescence kinetics}

In general, the growth mode of an epilayer is determined by both bulk thermodynamics and surface kinetics. However, in the nonequilibrium conditions characteristic for III-nitride growth, surface kinetics plays often a dominant role, especially during the early stages of nucleation and growth. Using a Peclet number (the ratio between the system length and the diffusion length) ${ }^{39,40}$
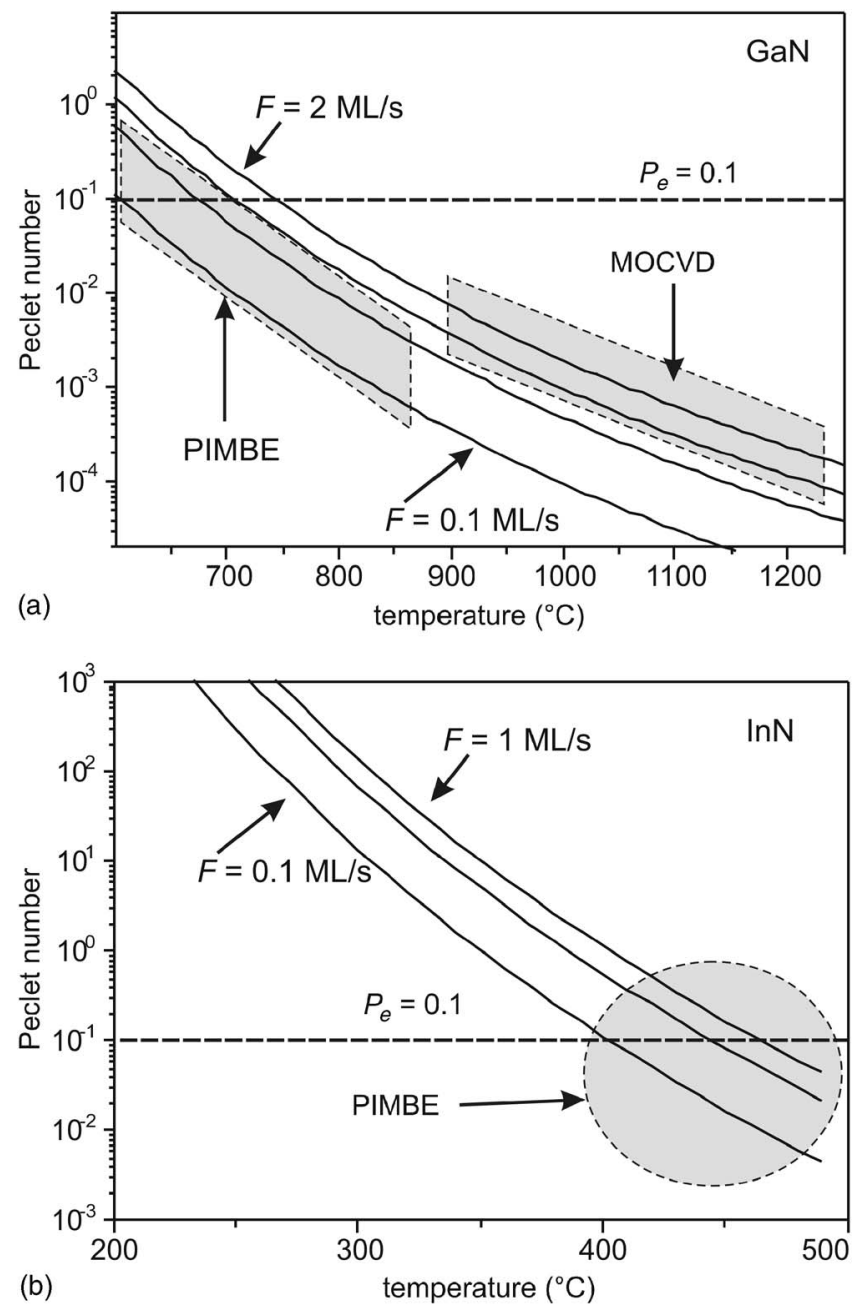

FIG. 8. The Peclet number as a criterion of the rapid coalescence process for (a) PIMBE and MOCVD of GaN and (b) PIMBE of InN.

$$
P_{e}=\frac{\tau_{\text {diffusion }}}{\tau_{\text {arrival }}}=\frac{L^{2} F}{D_{s}},
$$

as a criterion, we can define the growth conditions for a required rapid coalescence $\left(P_{e} \ll 1\right)$. For $P_{e}>1$, the surface mobility is low, promoting sticking of the species on top of the islands. As an example, we can consider the MOCVD GaN nanostripes discussed in the experimental part (Fig. 3). For this structure, $L \sim 50 \mathrm{~nm}$ is the mean distance between nucleation sites on the surface and $F$ is the arrival rate of the species on the surface $\left(\sim 2 \mathrm{ML} \mathrm{s}^{-1}\right.$ for the MOCVD growth rate $\left.\sim 16 \mathrm{~nm} \mathrm{~min}^{-1}\right)$. In this particular case, $P_{e} \sim 0.002 \ll 1$ indicates that the surface diffusion is a dominant process providing a high rate of the lateral growth of initial nuclei.

The Peclet number as a function of GaN growth temperature is shown in Fig. 8(a). One can see that conditions for the rapid coalescence for MOCVD growth are rather easily achievable than those for the case of PIMBE. It leads to a higher characteristic domain size during MOCVD growth usually resulting in a better device performance. Similar consideration performed for the InN epilayer PIMBE growth [see Figs. 5(a) and 5(b)] gives principally different results. Due to the low diffusion at the characteristic growth temperature $\sim 370-400{ }^{\circ} \mathrm{C}$, the condition $P_{e} \sim 0.1$ can be ful- 
filled only at low growth rates of $0.1-0.5 \mathrm{ML} \mathrm{s}^{-1}$ [Fig. 8(b)]. However, for the specific conditions of the $\mathrm{InN}$ heteroepitaxy, the high value of $P_{e}$ cannot be considered as a disadvantage. Indeed, the physical meaning of the Peclet number for the nucleation is also the island density, $N$, which is a function of the atomic arrival flux $F\left(\mathrm{ML} \mathrm{s}^{-1}\right)$ and $D_{s}(T)$. Employing rate equations and using the analytical approximation suggested by Mandreoli et al., ${ }^{7}$ we can describe the slope of $N\left(F / D_{s}\right)$ by the equation $N \cong\left(F / D_{s}\right)^{1 / 3}$ and, thus, get $P_{e} \propto N^{3}$. Thus, with increasing $F / D_{s}$ ratio, the island density rapidly increases, and for very high $F / D_{s}$ ratios, the adatom diffusivity is negligible and nuclei are no longer formed by two adatoms moving together but by adsorption of one adatom next to another existing adatom. ${ }^{7}$ Under these circumstances, a rapid coverage of the substrate surface by InN nuclei can be achieved at low temperatures.

As we discussed, the density and size of the growing islands are mainly determined by the balance between the rates of adsorption and diffusion of group-III atoms on the sample surface. As it follows from Eq. (5), if adsorption occurs slowly compared to the surface diffusion $\left(\tau_{\text {diffusion }}\right.$ $\ll \tau_{\text {arrival }}$, the surface approaches near thermal equilibrium at each moment during the growth resulting in low densities and large diameters of islands. ${ }^{17}$ In this case, the growth is governed mainly by kinetics. This situation is more peculiar for MOCVD growth due to higher surface temperatures and, consequently, higher $D_{s}$. For the case of PIMBE, the adsorption is much faster compared to the diffusion resulting in $P_{e} \sim 1$, so atoms tend to aggregate into small nuclei resulting in a higher density of domains for the same amount of adsorbed material $\left[\mathrm{N} \propto\left(P_{e}\right)^{1 / 3}\right]$.

The presented estimations, however, assume a serious simplification of the real processes. The presence of various structural features (i.e., steps, kinks, and facets) on the nuclei surfaces results in longer coalescence times due to edge barriers. ${ }^{7,41}$ At long time scales the presence of facets can substantially slow down surface diffusion while the model calculation in Eq. (5) assumes the particles to be perfectly spherical. Moreover, the surface diffusion is not the only operative process and, for instance, Ostwald ripening (a smaller particle is absorbed by a larger one) has to be also taken into account at high temperatures. ${ }^{17,42}$

Another example compares the coalescence occurring during PIMBE of GaN LPHs. We have previously demonstrated from RHEED and HRTEM analyses that Ga-face GaN grows on AlN elastically accommodating a misfit strain of $2 \%$, while $\mathrm{N}$-face $\mathrm{GaN}$ on sapphire should accommodate a large initial misfit of $16 \%$ by introducing GMDs ${ }^{12}$ resulting in a residual misfit $\sim 2 \%$. In both cases, however, the conditions for $2 \mathrm{D}$ growth are not fulfilled due to the residual stress and a relatively low nucleation temperature $\sim 650{ }^{\circ} \mathrm{C}$ resulting either in the Volmer-Weber or Stranski-Krastanow growth modes.

For GaN LPHs, under circumstances of nearly identical growth conditions the Peclet number criteria might be used to describe the differences in the coalescence occurring for Ga- and $\mathrm{N}$-face materials. In the ideal case $\left(P_{e} \ll 1\right)$, island sizes increase rapidly with increasing the amount of deposited material $\left(\langle A\rangle \sim t^{k}\right),{ }^{13}$ up to a coalescence point. As the thickness increases further, there is a progressive lateral coalescence of islands, which, thus, become definitely elongated until forming an almost perfect layer. In this mode the coalescence is completed for the Ga-face material, while $\mathrm{N}$-face stripes are noncontinuous films consisting of hexagonshaped domains as shown in Fig. 1(b). Taking into account the Peclet criteria, the following implication can be written for the Ga- and N-faces of GaN LPH:

$$
\left(P_{e}^{\mathrm{Ga}}=\frac{L^{2} F}{D_{s}^{\mathrm{Ga}}} \ll P_{e}^{\mathrm{N}}=\frac{L^{2} F}{D_{s}^{\mathrm{N}}}\right) \Rightarrow D_{s}^{\mathrm{Ga}} \gg D_{s}^{\mathrm{N}} .
$$

The condition $D_{s}^{\mathrm{Ga}} \gg D_{s}^{\mathrm{N}}$ correlates well with theoretical calculations $5,21,27,28$, where it was shown that the surface diffusivity is significantly higher on Ga face compared to the $\mathrm{N}$ face. This fact was attributed to the very different atomic geometries of the polar surfaces, where each surface atom of the $(000 \overline{1})$ surface has three dangling bonds compared to only one at the (0001) surface. Taking into account the calculated diffusion barriers ${ }^{5}$, the ratio between the diffusion coefficients for $\mathrm{Ga}$ atoms on $\mathrm{Ga}$ and $\mathrm{N}$ surfaces (and consequently for the Peclet number) can $\sim 10^{3}$.

In general, the balance between adsorption and diffusion can be modified by decreasing the growth rate and/or the substrate temperature or by increasing the V/III ratio, which leads to a lower diffusion rate. However, optimal growth conditions for both polarities within one structure are rather difficult to achieve. The $\mathrm{N}$-face islands being in contact with the sapphire substrate remain rather immobile, which can be explained also from the fact that their adhesion to the substrate is larger due to possible partial submersion. ${ }^{17}$ This can be explained by the difference in the surface energy of $\operatorname{GaN}(0001) \gamma=1.89 \mathrm{~J} / \mathrm{m}^{2}$ and the sapphire substrate, i.e., $\gamma$ $=1.47 \mathrm{~J} / \mathrm{m}^{2}$. As we have demonstrated in the experimental part, the growth of a low-temperature buffer layer is the only solution possible for this particular case.

\section{Domain boundaries on $\{11 \overline{20}\}$ and $\{10 \overline{0} 0\}$ planes}

As a consequence of the hexagonal symmetry of the epilayers, two kinds of domain boundaries (DBs) can be formed during the coalescence lying on the $\{11 \overline{2} 0\}$ and $\{10 \overline{1} 0\}$ planes. These two types of the domain boundaries in $2 \mathrm{H}$ structures have been studied experimentally by Xin $e t$ $a{ }^{10}{ }^{10}$ using $\mathbf{g} \cdot \mathbf{R}$ and HRTEM analyses and theoretically by Northrup et ll $^{43}$ by first principles calculations. In these works, both noncoherent $\{1 \overline{2} 10\}$ and $\{1 \overline{1} 00\}$ boundaries were considered to be of the stacking mismatch boundary (SMB) type. It was suggested that the $\{1 \overline{2} 10\}$ boundary, having a displacement of magnitude and direction $\frac{1}{2}\langle 10 \overline{1} 1\rangle$, arises from the coalescence of two islands, one of which is associated with a single growth fault on the basal plane. The $\{1 \overline{1} 00\}$ boundary has been proposed to originate at the epilayer/substrate interface and runs through the whole epilayer with a displacement of $1 / 3 n\langle 11 \overline{2} 0\rangle(n<3)$ in the basal plane and with an additional shift along $\langle 0001\rangle$, although the exact magnitude of the displacement of this boundary was not considered. Additionally, Xin et al. ${ }^{10}$ defined two pos- 

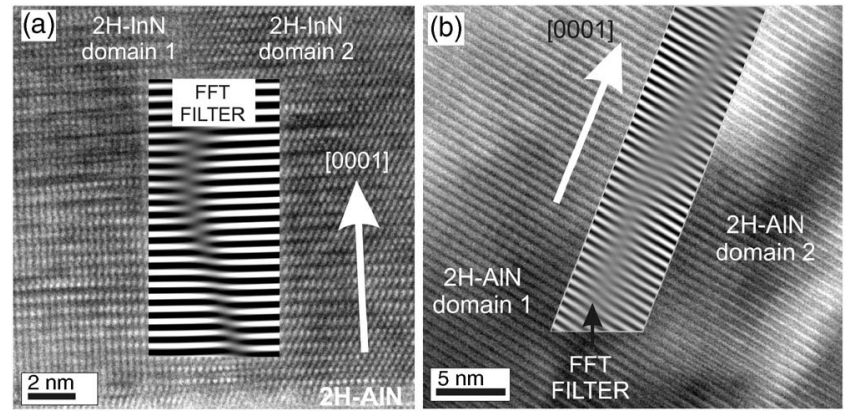

FIG. 9. HRTEM image registered along the $<11 \overline{2} 0>$ zone axis of a $2 \mathrm{D}$ DB between (a) two $2 \mathrm{H}$-InN domains placed on AlN template and (b) two $2 H$-AlN domains placed on $3 C$-SiC $(111) / \mathrm{Si}(111)$ template; Insets: FTfiltered area of SMB showing more clearly the disregistry between pairs of (0001) plane of the $2 H$ structure in each domain.

sible kinds of SMBs, one consisting of a different stacking sequence across the boundary, and the other being an inversion domain boundary (IDB) which is characterized by a polarity inversion across the boundary. The latter is also characteristic for highly faulted materials.

An example of a self-formed, IDB-containing structure are those $\mathrm{AlN}(0001)$ layers nucleated on $(1 \times 1) \mathrm{Si}(111)$ surfaces at high temperatures exhibiting a bipolar surface as proven by x-ray photoelectron diffraction. ${ }^{32}$ In this context, IDBs are of practical importance in LPHs, ${ }^{22}$ where the periodical change in the polarity can be used to achieve nonlinear optical effects as, for example, second harmonic generation. The structural properties of the IDBs are out of the scope of this work and will be discussed elsewhere. However, note that Northrup et al $^{43}$ calculated the domain wall energies for $\{1 \overline{1} 00\}$ IDBs in $2 H-G a N$ by first principles methods and demonstrated that the IDB with a $c / 2$ translation along (0001) has a low energy due to the fourfold coordination exhibited by its atoms, thus prohibiting the formation of wrong bonds (i.e., $\mathrm{Ga}-\mathrm{Ga}$ and $\mathrm{N}-\mathrm{N}$ ).

The theoretical ${ }^{43,44}$ and phenomenological ${ }^{10}$ SMB models are based on a stacking fault (SF) in the basal plane, which terminates at the boundary plane and results in a different stacking sequence across the boundary. Such disregistries are highlighted in Figs. 9 and 10(a), where the details of the Fourier transform (FT) filtered HRTEM micrograph show the shift between (0001) basal planes approaching both sides of the SMB planes in the $2 H$-InN epilayers. A general model suggests a single growth fault on the basal plane of one island exhibiting a displacement relative to an unfaulted island. Hence, the SMB has a displacement along $\langle 0001\rangle$ of $1 / n\langle 0001\rangle \quad(n>2)$ and a displacement of a fraction of $1 / 3\langle 11 \overline{2} 0\rangle$ within the boundary plane. Because basal plane stacking faults are of low energy, they were proposed as the origin of SMBs.

Here, we propose a simple growth model to explain the formation of SMBs formed during the coalescence. The nucleation of the highly mismatched $2 \mathrm{H}$-III-nitride material leads to the growth of islands having a slight deviation in the $c$-axis orientation. Earlier, we have demonstrated that tilt and twist of the simultaneously growing domains, with respect to each other and to the $c$ axis of the substrate, is characteristic
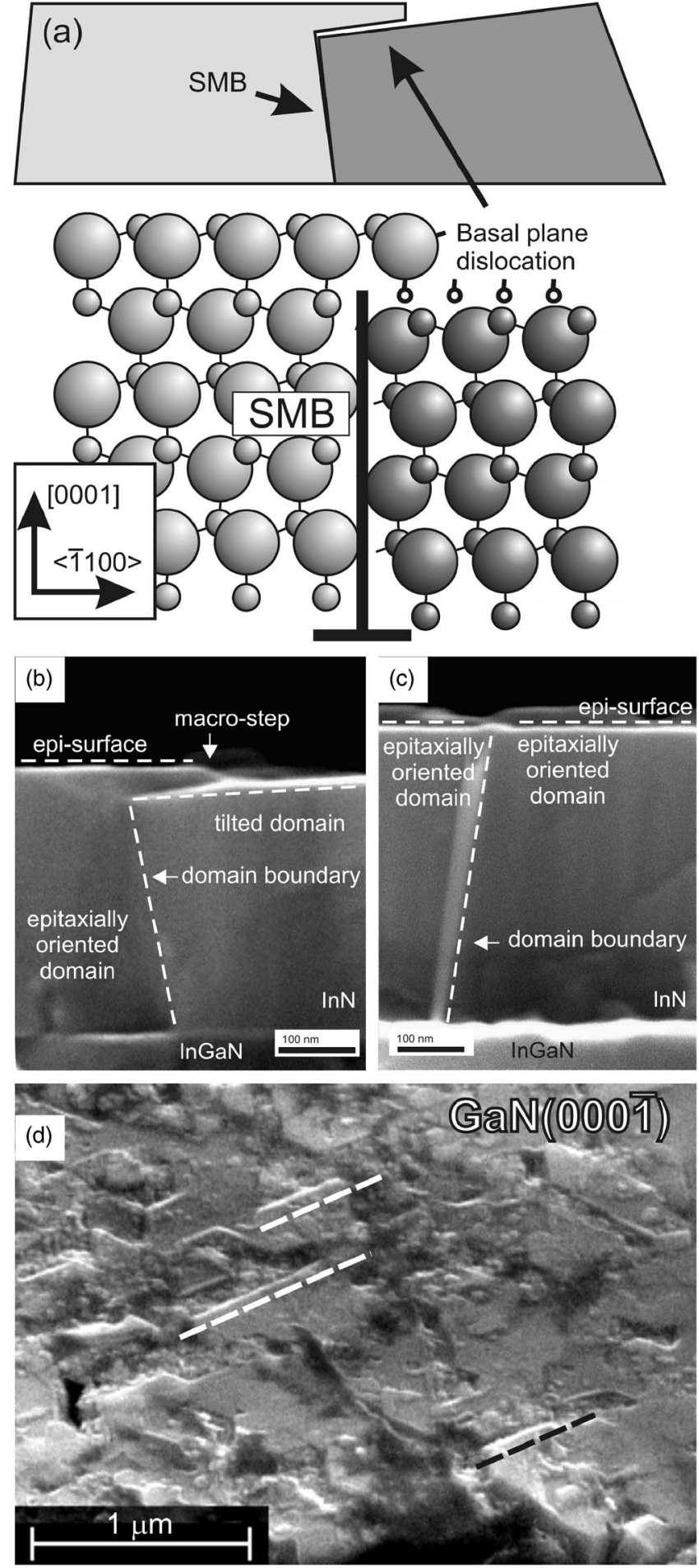

FIG. 10. (a) Atomic plane arrangement at the SMB region; schematic representation of the formation mechanisms of a low-angle SMB and a basal plane dislocation as a result of the coalescence process; [(b) and (c)] crosssection SEM images of the InN layer show (b) a lateral interaction of the epitaxial and tilted domains resulting in a characteristic surface macrostep, and (b) a completed coalescence of two epitaxially oriented domains; (d) SEM image of $\mathrm{N}$-face $\mathrm{GaN} / \mathrm{sapphire}$ structure demonstrating formation of surface steps (highlighted by the dashed lines) induced by the coalescence of the tilted domains.

for highly mismatched systems with a mismatch $f>10 \%$; e.g., $2 H$-AlN/Si (Refs. 33 and 34 ) and $2 H$-InN/AlN. ${ }^{12}$ The magnitude of the tilt depends on the magnitude of the epilayer/substrate mismatch and on actual growth conditions. 
It was also suggested that the origin of the tilt on the (0001) basal plane is a partial accommodation of the residual mismatch in certain crystallographic directions. On the other hand, in the nearly coincident systems such as $\mathrm{AlGaN} / \mathrm{GaN}$ such tilt and twist deviations have been rarely reported.

In Fig. 9, a HRTEM micrograph of two slightly tilted $2 \mathrm{H}$-InN domains ( 1 and 2$)$ is presented. They form a lowangle $\left(\sim 0.9^{\circ}\right.$ between $\langle 0001\rangle$ directions) noncoherent SMB along the $\{1 \overline{1} 00\}$ planes. As commented before, the displacement among (0001) planes is highlighted by the FT filtering. Similarly to the work by Xin et al., the magnitude of the local displacement vector along $\langle 0001\rangle$ does not exceed $1 / 2\langle 0001\rangle$. The maximum vertical displacement between the topmost (0001) planes of two domains in contact, $b$, can be calculated using a tilt angle $\alpha$ and a domain radius $d$ $\sim 100 \mathrm{~nm}$ as $b \sim d \tan \alpha=1.57 \mathrm{~nm}$.

A high magnitude of the total displacement has two important consequences for the coalescence. Firstly, tilted domains form a noncoherent boundary along the $\{1 \overline{1} 00\}$ plane. Additionally, the local displacement of the (0001) planes is not constant, but does not exceed $c / 2$. Secondly, as it is shown in Fig. 10, the interaction of a tilted terminating (0001) plane with the wall of a neighboring domain, which is normally oriented to the substrate [(0001) sapphire// $\left.(0001)_{\text {domain }}\right]$, induces the formation of a surface macrostep by the lateral growth of the normally oriented domain [shown in Figs. 10(b)-10(d)]. Thus, the appearance of a basal dislocation with $\mathbf{b}=1 / 3\langle 11 \overline{2} 0\rangle$ can be associated with the formation of the predicted (0001) SF. The possible termination of TDs induced by the coalescence of the in-plane misoriented (twisted) $2 H$ domains ${ }^{11,12}$ could be then explained here as the stopping, bending, propagation, and annihilation of TDs.

Despite the fact that $\mathbf{b}=1 / 3\langle 11 \overline{2} 0\rangle$ basal dislocations have a relatively low density of $\sim 10^{8} \mathrm{~cm}^{-2}$, they play an important role in the elimination of TDs in the $2 \mathrm{H}$ epilayer. ${ }^{12}$ The latter is illustrated by the TEM micrographs shown in Figs. 11(a) and 11(b), where the TD density is shown to decrease drastically from the near interface region towards the AlN or InN surfaces, respectively. As it is summarized in Fig. 11(c), our annihilation model assumes the interaction of $\mathbf{b}=1 / 3\langle 11 \overline{2} 0\rangle$ mismatch TDs and basal plane SFs at the vicinity of domain edges along the $\{1 \overline{1} 00\}$ planes.

\section{CONCLUSIONS}

In conclusion, we have examined the coalescence phenomena in thin binary $2 \mathrm{H}$-III-nitride epilayers by means of electron and atomic force microscopies and of x-ray and electron diffractions. This study generalizes the growth parameters responsible for the rapid coalescence of III-nitride films, and describes the coalescence qualitatively and, where possible, analytically for the case of heteroepitaxy in the nonequilibrium conditions. Coalescence time and the corresponding diffusion coefficients at elevated temperatures were estimated for $2 \mathrm{H}$-GaN and $2 \mathrm{H}$-InN growths. The rate of coalescence has been found to impact the structure and morphology of III-nitride epilayers.
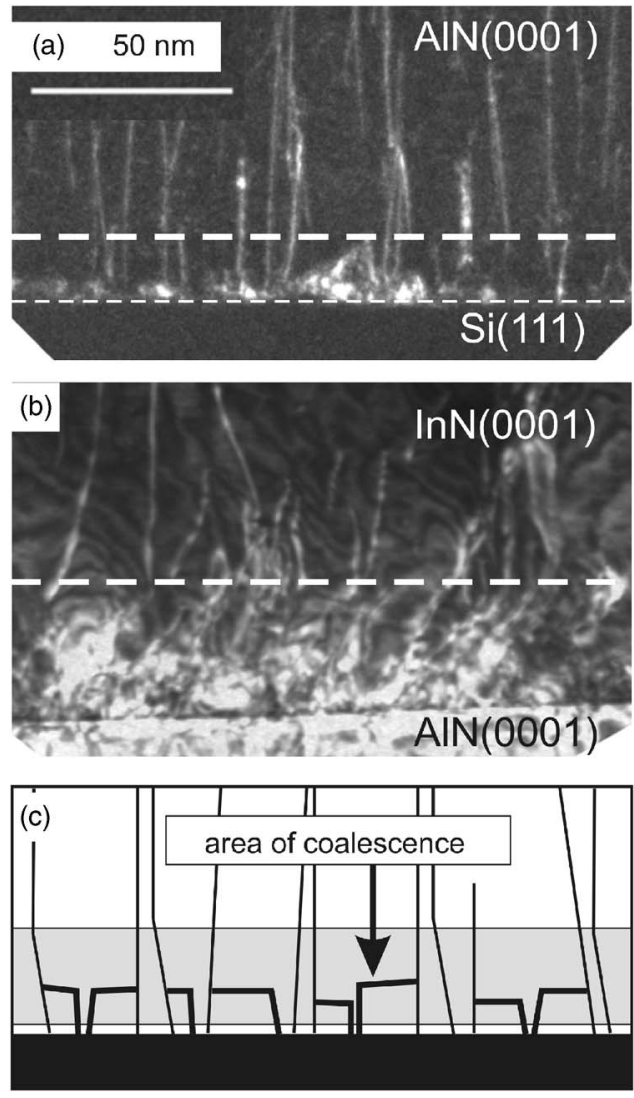

FIG. 11. [(a) and (b)] 2B-DF-XTEM micrographs of $\mathrm{AlN}(0001) / \mathrm{Si}(111)$ and InN/AlN(0001) heterostructures demonstrate a highly faulted region near the heterointerface. (c) The annihilation model proposes that the TD density is partially reduced due to the interaction with basal plane dislocations formed during the coalescence of domains.

Additionally, we suggest a simple growth model to explain the formation of the domain boundaries and (0001) stacking faults formed during the coalescence. In particular, it was shown experimentally that tilted, hexagonal-shaped $2 \mathrm{H}$ domains form a noncoherent boundary along the $\{1 \overline{1} 00\}$ plane. Moreover, the local displacement of the (0001) planes was found to be variable but not exceeding $c / 2$. We also suggest that the interaction of tilted domains induces a basal dislocation to appear and annihilate due to the formation of a (0001) stacking fault. It was demonstrated that this interaction has two obvious consequences: a lateral growth of the most normally oriented domains followed by the formation of a surface step, and consequently the termination of TDs induced by the coalescence.

\section{ACKNOWLEDGMENTS}

This work was supported by DFG Grant No. AM105/1-1 (Germany), CICYT Project No. MAT2004-01234 (Spain), SANDIE European Network of Excellence (NMP4-CT2004-500101-Sixth Framework Program), and by ONR NICOP Grant No. 00014-03-1-0301. One of the authors (F.M.M.) would like to thank the Alexander von Humboldt Foundation for the financial support under a Humboldt Research Fellowship SPA/1114640STP. 
${ }^{1}$ M. Henini and M. Razeghi, Optoelectronic Devices: III Nitrides (Elsevier, New York, 2005).

${ }^{2}$ C. G. Van de Walle and J. Neugebauer, J. Appl. Phys. 95, 3851 (2004).

${ }^{3}$ M. A. Reshchikov and H. Morkoç, J. Appl. Phys. 97, 061301 (2005).

${ }^{4}$ R. M. Feenstra, Y. Dong, C. D. Lee, and J. E. Northrup, J. Vac. Sci. Technol. B 23, 1174 (2005).

${ }^{5}$ T. Zywietz, J. Neugebauer, and M. Scheffler, Appl. Phys. Lett. 73, 487 (1998).

${ }^{6}$ J. Neugebauer, T. Zywietz, M. Scheffler, J. Northrup, H. Chen, and R. M. Feenstra, Phys. Rev. Lett. 90, 056101 (2003).

${ }^{7}$ L. Mandreoli, J. Neugebauer, R. Kunert, and E. Schöll, Phys. Rev. B 68, 155429 (2003).

${ }^{8}$ N. Takeuchi, A. Selloni, T. H. Myers, and A. Doolittle, Phys. Rev. B 72, 115307 (2005).

${ }^{9}$ W. Qian, M. Skowronski, M. De Graaf, K. Doverspike, L. B. Rowland, and D. K. Gaskill, Appl. Phys. Lett. 66, 1252 (1995).

${ }^{10}$ Y. Xin, P. D. Brown, and C. J. Humphreys, Appl. Phys. Lett. 70, 1308 (1997).

${ }^{11}$ A. Bourret, C. Adelmann, B. Daudin, J. Rouvière, G. Feuillet, and G. Mula, Phys. Rev. B 63, 245307 (2001).

${ }^{12}$ V. Lebedev et al., J. Appl. Phys. 100, 094902 (2006).

${ }^{13}$ A. J. Bray, Adv. Phys. 43, 357 (1994).

${ }^{14}$ D. Weaire and S. McMurry, Solid State Phys. 50, 1 (1997)

${ }^{15}$ A. Kazaryan, Y. Wang, S. A. Dregia, and B. R. Patton, Phys. Rev. B 61, $14275(2000)$

${ }^{16}$ A. Kazaryan, Y. Wang, S. A. Dregia, and B. R. Patton, Phys. Rev. B 63, 184102 (2001).

${ }^{17}$ G. Palasantzas, T. Vystavel, S. A. Koch, and J. Th. M. De Hosson, J. Appl. Phys. 99, 024307 (2006).

${ }^{18} \mathrm{~K}$. Tonisch, V. Cimalla, Ch. Förster,H. Romanus, O. Ambacher, and D. Dontsov, Sens. Actuators, A 132, 658 (2006).

${ }^{19} \mathrm{~K}$. Tonisch, V. Cimalla, F. Will, F. Weise, M. Stubenrauch, A. Albrecht, M Hoffmann, and O. Ambacher, Physica E (Amsterdam) (2006), available online (doi: 10.1016/j.physe.2006.06.002

${ }^{20}$ V. Lebedev, V. Cimalla, U. Kaiser, Ch. Foerster, J. Pezoldt, and O. Ambacher, J. Appl. Phys. 97, 114306 (2005).

${ }^{21}$ C. Adelmann, J. Brault, G. Mula, B. Daudin, L. Lymperakis, and J. Neugebauer, Phys. Rev. B 67, 165419 (2003).

${ }^{22}$ P. J. Schuck, M. D. Mason, R. D. Grober, O. Ambacher, A. P. Lima, C.
Miskys, R. Dimitrov, and M. Stutzmann, Appl. Phys. Lett. 79, 952 (2001).

${ }^{23}$ I. Cimalla, Ch. Foerster, V. Cimalla, V. Lebedev, D. Cengher, and O. Ambacher, Phys. Status Solidi C 3, 1767 (2006).

${ }^{24}$ W. W. Mullins, J. Appl. Phys. 28, 333 (1957).

${ }^{25}$ J. E. Northrup and J. Neugebauer, Phys. Rev. B 53, R10477 (1996).

${ }^{26}$ J. E. Northrup, L. T. Romano, and J. Neugebauer, Appl. Phys. Lett. 74, 2319 (1999).

${ }^{27}$ B. Heying, R. Averbeck, L. F. Chen, E. Haus, H. Riechert, and J. S. Speck, J. Appl. Phys. 88, 1855 (2000).

${ }^{28}$ J. Neugebauer, T. Zywietz, M. Scheffler, J. Northrup, H. Chen, and R. M. Feenstra, Phys. Rev. Lett. 90, 056101 (2003).

${ }^{29}$ J. Narayan, K. Dovidenko, A. Sharma, and S. Oktyabrsky, J. Appl. Phys. 84, 2597 (1998).

${ }^{30}$ K. Dovidenko, S. Oktyabrsky, J. Narayan, and M. Razeghi, J. Appl. Phys. 79, 2439 (1996).

${ }^{31}$ S. Yoshida, S. Misawa, and S. Gonda, Appl. Phys. Lett. 42, 427 (1983).

${ }^{32}$ V. Lebedev, B. Schröter, G. Kipshidze, and W. Richter, J. Cryst. Growth 207, 266 (1999).

${ }^{33}$ V. Lebedev, J. Jinschek, J. Kräußlich, U. Kaiser, B. Schröter, and W. Richter, and J. Cryst. Growth 230, 430 (2001).

${ }^{34}$ V. Lebedev, J. Jinschek, U. Kaiser, B. Schröter, W. Richter, and J. Kräußlich, Appl. Phys. Lett. 76, 2029 (2000).

${ }^{35}$ X. Ning, F. R. Chien, P. Pirouz, J. W. Yang, and M. Asif Khan, J. Mater. Res. 11, 580 (1996).

${ }^{36} \mathrm{~V}$. Lebedev and W. Richter, in Vacuum Science and Technology: Nitrides as Seen by the Technology, edited by T. Paskova and B. Monemar, (Research Signpost, Kerala, 2002).

${ }^{37} \mathrm{~J}$. Howe, Interfaces in Materials (Wiley, New York, 1997).

${ }^{38}$ R. Kern and P. Miller, Surf. Sci. 392, 103 (1997).

${ }^{39}$ P. Molho, A. J. Simon, and A. Libchaber, Phys. Rev. A 42, 904 (1990).

${ }^{40}$ J. Tsao, Materials Fundamentals of Molecular Beam Epitaxy (Academic, San Diego, CA, 1993).

${ }^{41}$ L. Lewis, P. Jensen, and J.-L. Barrat, Phys. Rev. B 56, 2248 (1997).

${ }^{42}$ F. Haußer and A. Voigt, Phys. Rev. B 72, 035437 (2005).

${ }^{43}$ J. E. Northrup, J. Neugebauer, and L. T. Romano, Phys. Rev. Lett. 77, 103 (1996).

${ }^{44}$ J. Elsner, M. Kaukonen, M. I. Heggie, M. Haugk, Th. Frauenheim, and R. Jones, Phys. Rev. B 58, 15347 (1998). 